\title{
Depression of Activity in the Corticospinal Pathway during Human Motor Behavior after Strong Voluntary Contractions
}

\author{
Nicolas T. Petersen, Janet L. Taylor, Jane E. Butler, and Simon C. Gandevia \\ Prince of Wales Medical Research Institute and University of New South Wales, Sydney, Australia 2031
}

The corticospinal system plays an important role in control of voluntary movements in primates. Recently, we demonstrated that the effectiveness of this system is depressed after maximal exercise. Because the depression was absent after antidromic activation of the motoneurons, we argued that transmission across corticospinal synapses was involved. Here, we explore the possible functional consequences of such a depression. In humans, direct electrical stimulation of axons of corticospinal neurons at the cervicomedullary level evokes motor potentials in elbow flexor muscles. When tested during relaxation after a maximal voluntary contraction (MVC) of the elbow flexors, potentials in biceps brachii and brachioradialis were depressed for $\sim 90 \mathrm{sec}$. The potentials were also depressed, although less markedly, when tested during a weak elbow flexion. Brief intermittent MVCs abolished the depression transiently, but during the intervening periods of relaxation, the depression appeared similar to that during continuous relaxation. The depression was greatest during relaxation after a $10 \mathrm{sec} \mathrm{MVC}$ and smaller after submaximal contractions. To look for effects of the depression on voluntary activity, we compared bilateral matching weak elbow flexions. After a conditioning $10 \mathrm{sec}$ maximal elbow flexion of one arm, the electromyographic activity produced on that side was reduced relative to the activity on the contralateral side. Our findings support the view that synapses in the corticospinal system are depressed after strong voluntary contractions during both relaxation and activity. Furthermore, this depression can affect the production of voluntary movement.

Key words: corticospinal tract; motor cortex; transmastoid stimulation; electrical stimulation; synaptic depression; human

\section{Introduction}

Neural activity produces variable potentiation and depression at most synapses, with much of this plasticity resulting from altered synaptic efficacy (Zucker, 1989; Fisher et al., 1997; Wu and Saggau, 1997; Miller, 1998; Thomson, 2000; Zucker and Regehr, 2002). The primary motor cortex in primates provides a major corticospinal output through which voluntary movements are generated (Phillips and Porter, 1977; Porter and Lemon, 1993). In conscious nonhuman primates, the discharge frequency of corticospinal cells driving a muscle increases with the muscle force required (Evarts, 1968). When tested in anesthetized monkeys, the synaptic efficacy of corticospinal connections with motoneurons can show short-term changes (Porter, 1970; Muir and Porter, 1973; Shapovalov et al., 1978), although the underlying mechanisms have not been explored. In humans, the corticospinal system includes a direct monosynaptic route to proximal as well as distal muscles (de Noordhout et al., 1999; Petersen et al., 2002) and can be activated with single electrical stimuli at the cervicomedullary level (Ugawa et al., 1991; Gandevia et al., 1999). We showed previously that a sustained maximal voluntary contraction (MVC) of elbow flexor muscles induces an immediate

\footnotetext{
Received Sept. 9, 2002; revised May 28, 2003; accepted May 28, 2003.

This work was supported by the National Health and Medical Research Council of Australia Grant 3206. N.T.P. received support from the Weimann Foundation and the Danish Sports Research Council. We are grateful to Prof.S. Redman and Dr. J. Brock for their comments on this manuscript.

Correspondence should be addressed to Prof. Simon C. Gandevia, Prince of Wales Medical Research Institute, Barker Street, Randwick, New South Wales, Australia 2031. E-mail: s.gandevia@unsw.edu.au.

Copyright $\odot 2003$ Society for Neuroscience $\quad$ 0270-6474/03/237974-07\$15.00/0
}

reduction in the response of their motoneurons to electrical stimulation of the corticospinal tract (Gandevia et al., 1999). When the MVC was replaced by tetanic electrical stimulation of the motor nerves, the responses to corticospinal tract stimulation were not depressed. This lack of depression illustrates the need for corticospinal activation of the motoneurons and suggests that the corticospinal synapses are involved in the depression. However, the depression of responses to corticospinal tract stimulation was observed only after maximal contractions and only when subjects were tested during voluntary relaxation. This leaves open the questions of whether the depression can occur after weaker exercise or during activity and, thus, whether it may be of functional importance.

In the present study, we examined the responses to single corticospinal volleys after voluntary contractions to assess the intensity of exercise required for the depression of the efficacy of corticospinal transmission. We also examined whether the depression of these responses occurred during voluntary activity as well as during relaxation. Finally, we performed psychophysical experiments to evaluate how changes in the corticospinal system may influence normal motor activities.

Parts of this paper have been published previously in abstract form (Petersen et al., 2001a).

\section{Materials and Methods}

Subjects and experimental setup. Seven adult subjects participated in the experiments that involved different protocols in which the descending motor tracts were stimulated, and 10 naive additional subjects participated in psychophysical experiments that did not involve stimulation. All 
A

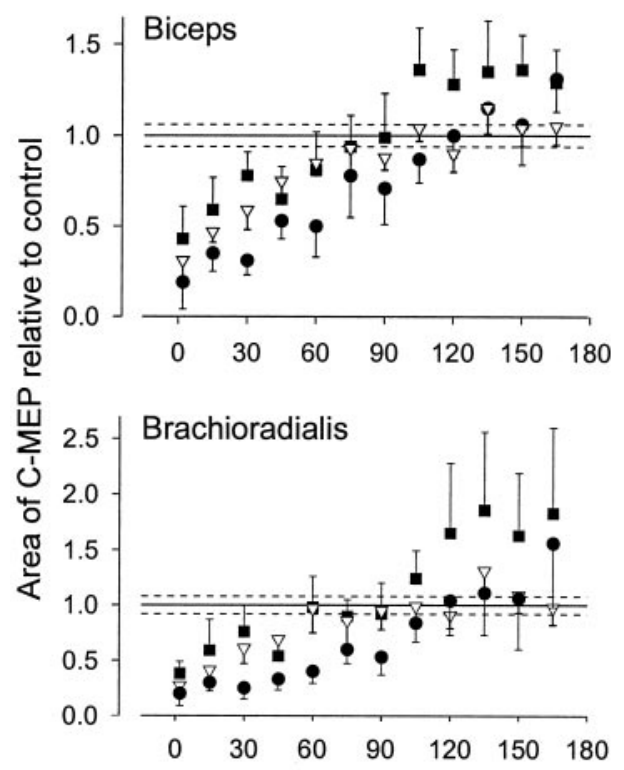

Time after conditioning contraction (s)

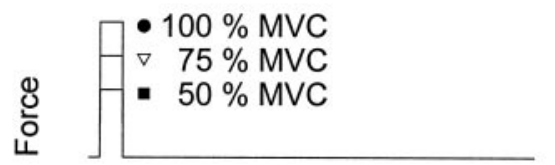

B

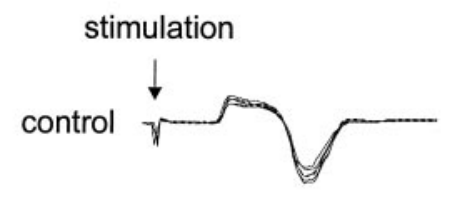

$10 \mathrm{~s}$ conditioning contraction

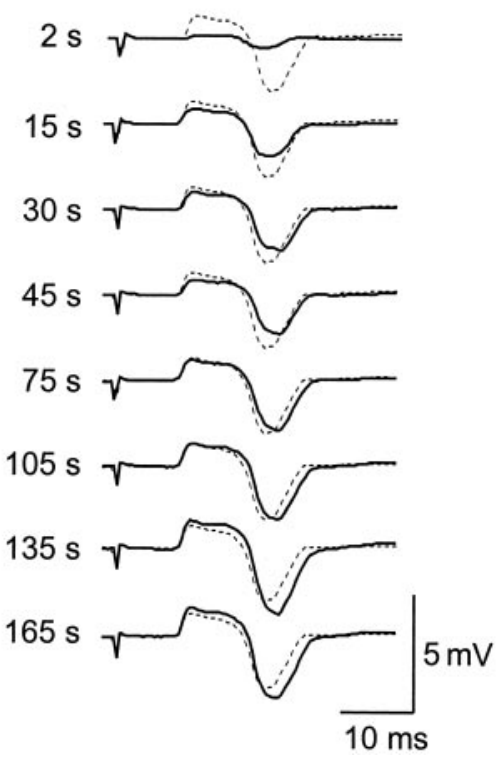

Figure 1. Changes in the responses to corticospinal stimulation (CMEPS) delivered at rest after voluntary contractions of three strengths. A, Data for biceps brachii (top), brachioradialis (middle), and the profile of voluntary forces (bottom). The area of the CMEPs was expressed relative to control values obtained before the conditioning contraction and is shown as the group mean and SEM. Timing of the conditioning contraction is shown at the bottom. Conditioning contractions were of three strengths: $50 \% \mathrm{MVC}$ (filled squares), 75\% MVC (open triangles), and 100\% MVC (filled circles). All responses were obtained with subjects relaxed. The solid and dotted horizontal lines indicate the mean \pm SEM of the control responses, respectively. Data were obtained in single sessions in five subjects. At least 45 min separated the contractions. The CMEPs are depressed after the 10 sec contractions, with the depression being greatest for the strongest contraction and least for the weakest one. ANOVA of responses in both muscles revealed significant depression for 90 sec after the $100 \% \mathrm{MVC}$, 45 sec after the $75 \% \mathrm{MVC}$, and 2 sec after the $50 \% \mathrm{MVC}(p<0.05)$. $B$, Individual traces from a single subject. Top traces are superimposed CMEPs in the control period before a $10 \mathrm{sec} 100 \%$ MVC. Traces below depict the CMEPs as a raster sequentially from 2 to 180 sec after the conditioning contraction. The broken lines depict the average control response.

subjects gave written informed consent to the experimental procedures, which had been approved by the local human research ethics committee. Subjects sat with one or both arms strapped in myographs with the forearm upright and the elbow and shoulder flexed to $90^{\circ}$. Elbow flexion forces could be measured and stored on computer. The elbow flexion force could be monitored by the subjects on an oscilloscope. When subjects made maximal efforts, they always received continuous verbal encouragement to obtain their maximal force.

Data acquisition. Electromyographic (EMG) activity was recorded from biceps brachii and brachioradialis using self-adhesive electrodes (1 $\mathrm{cm}$ in diameter). One electrode was placed over the midbelly of each muscle and the other over the tendon. EMG activity was filtered (16$1000 \mathrm{~Hz}$ ), digitized $(2 \mathrm{kHz})$, and stored on a computer (CED 1401 with Signal and Spike2 software; Cambridge Electronic Design, Cambridge, $\mathrm{UK})$.

Stimulation and stimulation protocols. Procedures for stimulation of the corticospinal tract at the cervicomedullary junction or the peripheral nerves at the brachial plexus were identical to those used previously (Gandevia et al., 1999; Taylor et al., 2000). In brief, the corticospinal tract was stimulated via surface electrodes fixed to the skin over the mastoid processes on each side (cathode on the left, $100 \mu \mathrm{sec}$ pulse Digitimer D180 or modified Digitimer DS7; Digitimer, Welwyn Garden City, UK) to evoke motor responses in the elbow flexors [termed cervicomedullary motor-evoked potentials (CMEPs)]. The brachial plexus was stimulated via a cathode in the supraclavicular fossa and an anode on the acromion $(100 \mu \mathrm{sec}$ pulse; Digitimer DS7). The areas of the CMEPs and M-waves were measured.

In the studies involving corticospinal stimulation, maximal voluntary elbow flexion force (MVC) and maximal muscle response $\left(M_{\max }\right)$ to electrical stimulation of the motor nerve at the brachial plexus were measured during the setup. The intensity of the electrical stimulation of the corticospinal tract was adjusted to produce CMEPs in biceps brachii of 25\% $M_{\text {max }}$, and this usually produced responses in brachioradialis of $\sim 5 \% M_{\max }$. A number of protocols involving stimulation of the corticospinal tract before and after conditioning contractions were used (see Figs. 1-3). Stimulation of the corticospinal tract was given at intervals ranging from 5 to $20 \mathrm{sec}$. The duration of the conditioning contraction was $10 \mathrm{sec}$ because MVCs of this duration produce depression of the response to corticospinal stimulation (at rest), and this depression is not augmented by longer contractions (Gandevia et al., 1999).

In the first study, subjects $(n=5)$ performed single conditioning contractions at three levels of maximal force in one experimental session (100\% MVC, $50 \%$ MVC, and then $75 \%$ MVC). A rest period of at least $45 \mathrm{~min}$ separated the contractions. Corticospinal stimuli were delivered before and at a series of intervals after each voluntary contraction. The size of the responses was expressed relative to that in the control period before each contraction.

In the second study, subjects $(n=4)$ again performed a $10 \mathrm{sec}$ conditioning contraction (100\% MVC) but in the control period before the MVC and also immediately afterward, subjects made a weak voluntary contraction at $\sim 5 \%$ MVC. For this, subjects received feedback of the integrated EMG activity from biceps brachii. This generated approximately the same output from the motoneurons before and after the conditioning contraction. Corticospinal stimuli were delivered during this weak activity before and after the conditioning contraction. In addition, supramaximal stimuli were delivered to the motor nerve throughout the experiment so that any change in the maximal muscle response could be assessed.

In the third study, subjects $(n=3)$ attended for two sessions separated by several days. In both, a $10 \mathrm{sec}$ conditioning MVC was used and corticospinal stimuli were delivered at rest before and after the main contraction (see Fig. 3A). However, in addition, responses were delivered during brief voluntary contractions (1-2 sec duration) before (in the control period) and after the conditioning MVC. In one session, the level of these brief contractions was $5 \% \mathrm{MVC}$, and, in the other, contractions were maximal (i.e., $100 \% \mathrm{MVC}$ ).

Psychophysical study. Subjects $(n=10)$ made weak bilateral contractions of the elbow flexors. They received force feedback from the left arm but were not told whether this feedback derived from the left or right side. Subjects were instructed to contract both arms simultaneously to reach a target force (10\% MVC) within $1 \mathrm{sec}$ after an auditory signal delivered at $5 \mathrm{sec}$ intervals. They were instructed not to correct contractions once initiated. After practice, subjects performed three sets of contractions. Each consisted of nine brief contractions, a $10 \mathrm{sec}$ rest, 18 brief contractions, a $10 \mathrm{sec}$ rest, 18 brief contractions, a $10 \mathrm{sec}$ MVC of the right arm 
A

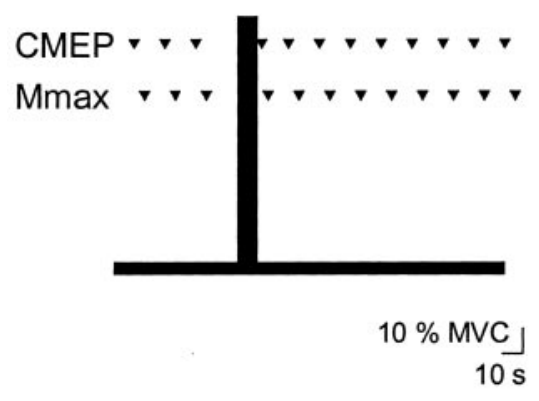

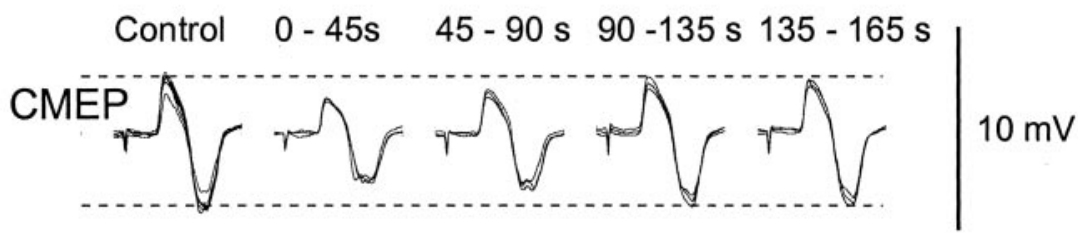
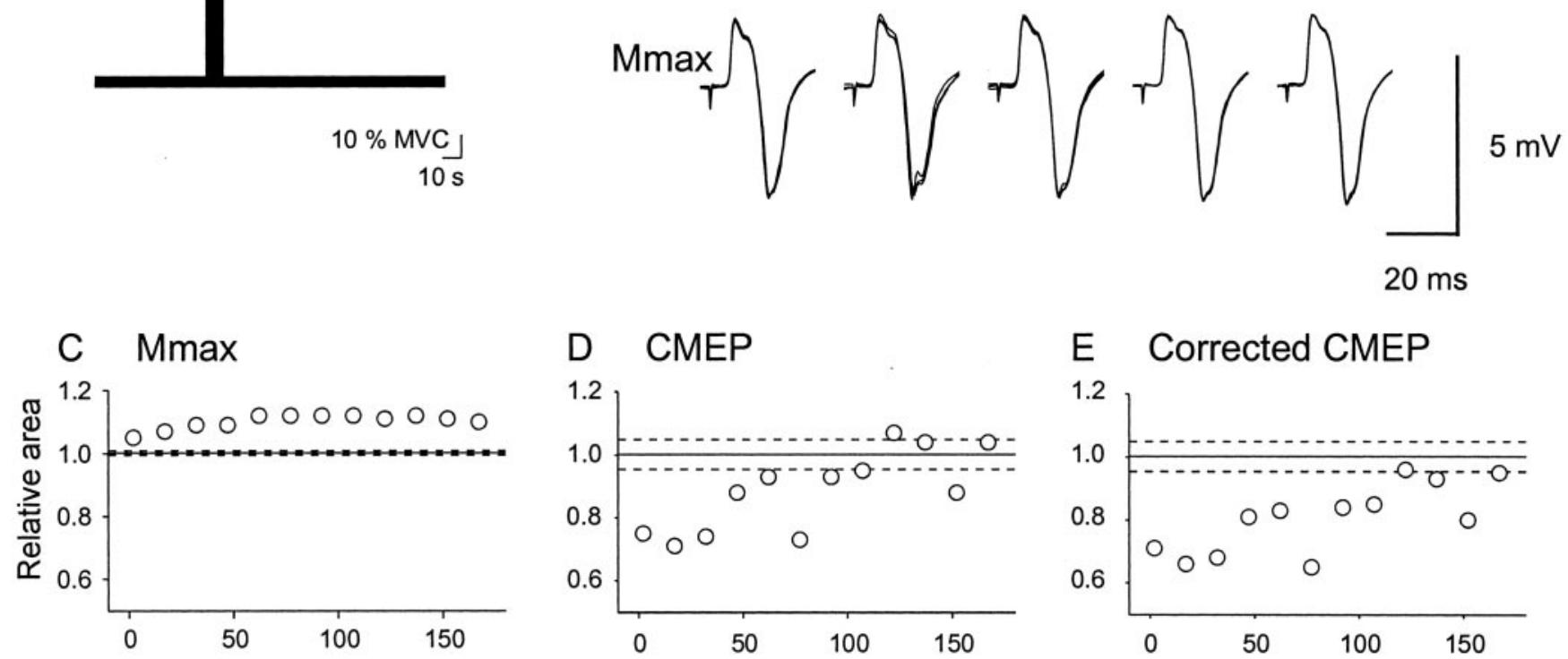

D CMEP

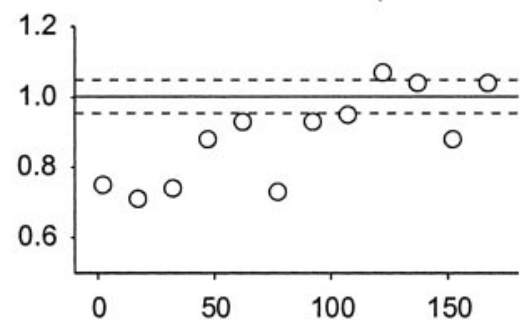

E Corrected CMEP

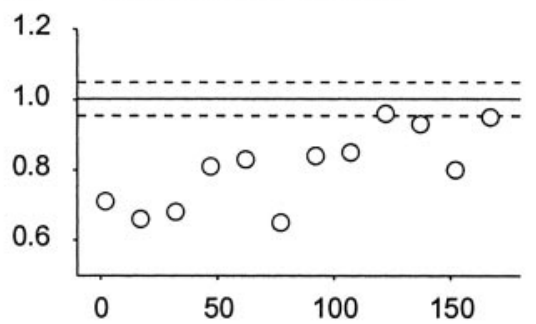

Time after conditioning contraction (s)

Figure 2. Responses from a single subject to corticospinal stimulation (CMEPs) after a $10 \mathrm{sec}$ MVC when the stimuli were delivered during a continuous weak voluntary contraction (5\% MVC). $A$, Experimental protocol. Corticospinal stimuli (CMEP) and maximal motor nerve stimuli $\left(M_{\max }\right)$ were delivered (arrowheads) during a 5\% MVC before and after a 10 sec conditioning MVC. $B$, Superimposed CMEPs and maximal motor responses $\left(M_{\max }\right.$, produced by stimulation of the motor nerve) recorded under control conditions before and after the conditioning MVC. C, Area of $M_{\max }$ plotted relative to initial control before the conditioning MVC. D, Area of the CMEP plotted relative to initial control before the conditioning MVC. E, Area of the CMEP corrected for the small increase in $M_{\max }$ shown in $B$. In $C-E$, the horizontal lines depict means \pm SEM for control responses.

only, and an additional nine brief contractions. Force and integrated EMG activity responses in the left and right arms were compared before and after the MVC of the right arm.

Analyses. Statistical analyses of CMEPs included ANOVA and Student-Newman-Keuls post hoc test. In the psychophysical experiment, EMG activity and force were analyzed using ANOVA on ranks and Dunnett's post hoc test.

\section{Results}

In the first study, we found that the long-lasting depression of the responses to corticospinal stimulation (CMEPs) in the relaxed elbow flexor muscles was influenced by the strength of the preceding $10 \mathrm{sec}$ conditioning voluntary contraction. Under control conditions, the amplitudes of CMEPs in biceps brachii and brachioradialis were 25 and 5\%, respectively, of the maximal responses to stimulation of the motor nerves. As shown in Figure 1, CMEPs in both muscles were depressed immediately after the voluntary contractions of $50-100 \%$ of maximal strength $(n=5$ subjects). In the first minute after the contractions, the depression was greater in magnitude with conditioning contractions of $100 \%$ MVC than 75 or $50 \%$ MVC $(p<0.05)$. It also lasted longer. ANOVA of responses in both muscles revealed significant depression for $90 \mathrm{sec}$ after the 100\% MVC, $45 \mathrm{sec}$ after the $75 \%$ MVC, and $2 \mathrm{sec}$ after the 50\% MVC ( $p<0.05)$. After this de- pression, a facilitation of the CMEP was sometimes observed (Fig. 1A,B) (see Fig. 3B).

We considered whether the depression of the CMEP might only occur after a voluntary contraction and thus not operate during normal movements. Therefore, in the second study, we examined the CMEP during a constant voluntary contraction. CMEPs elicited in biceps brachii were depressed while subjects $(n=4)$ maintained a steady level of EMG activity ( 5\% MVC) after a conditioning $10 \mathrm{sec}$ MVC (Fig. 2). This depression occurred despite a small increase in the response to stimulation of the motor nerves $\left(M_{\max }\right)$ (Fig. $2 B, C$ ) (Cupido et al., 1996; Gandevia et al., 1999). The depression of the CMEP during 5\% sustained contraction appeared less than in resting muscles, but direct comparison was not possible because the subjects were not the same in the two conditions.

In the third study, we compared the depression of the CMEP evoked during brief intermittent contractions (1-2 sec duration) with that of the CMEP evoked in resting muscle $4 \mathrm{sec}$ after each brief contraction in the same experiments (Fig. 3). In the control period, weak brief contractions ( $5 \% \mathrm{MVC}$ ) did not significantly influence subsequent CMEPs (Fig. $3 B$, precontrol vs control responses). When the brief contractions were strong (100\% MVC), the resting CMEPs were smaller than those in the precontrol 
A

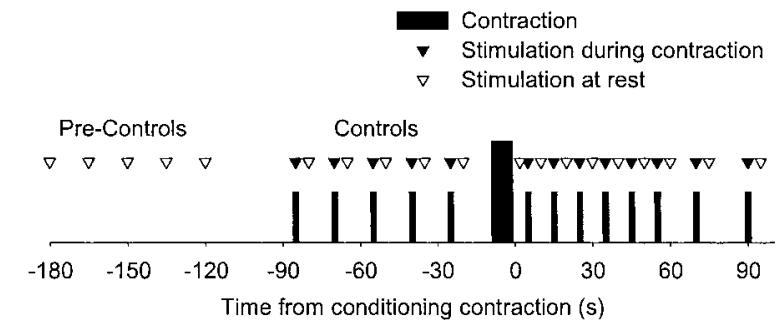

$\mathrm{B}$

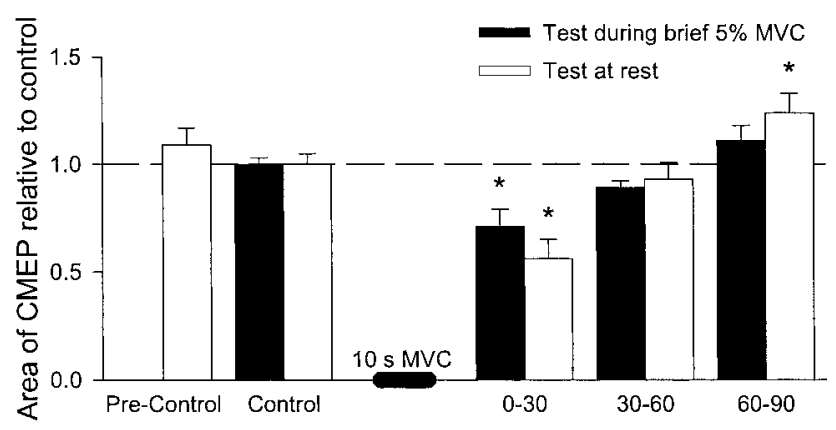

C

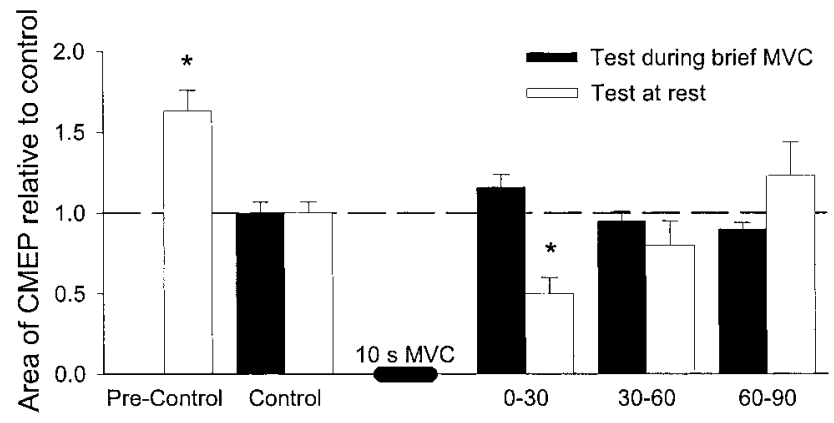

Figure 3. Responses in biceps to corticospinal stimulation (CMEPs) after a $10 \mathrm{sec}$ MVC when the stimuli were delivered at rest and also during brief voluntary contractions. $A$, Experimental protocol. Stimuli during rest were delivered initially (precontrols). Then, stimuli were delivered during brief voluntary contractions and at rest (controls). The subject next performed the usual 10 sec conditioning MVC, and responses were measured after it. We compare the postcontraction depression of the CMEPs measured during brief contractions at $5 \% \mathrm{MVC}(B)$ or $100 \% \mathrm{MVC}$ (C) with those obtained at rest. The CMEPs obtained in the 90 sec period after the conditioning contraction were grouped in 30 sec periods, and the size of each CMEP was expressed relative to that obtained in the control period immediately before the conditioning contraction. Responses obtained during a voluntary contraction are shown as filled symbols and those during rest as open symbols. Mean \pm SEM $(n=3)$. B, The brief $5 \%$ MVCs did not significantly alter the size of the following resting CMEPs. After the conditioning MVC, CMEPs were depressed when tested during contractions or rest. C, Before the conditioning contraction, there was some depression of the CMEPs because of the repeated brief MVCs, illustrated by the precontrols that have a value higher than one. However, after the conditioning contraction, the CMEPs elicited at rest were depressed and recovered over $\sim 60 \mathrm{sec}$, whereas the responses obtained during the brief intermittent MVCs were not depressed. ${ }^{*} p<0.05$, statistically significant differences from control.

period (Fig. 3C). After the 10 sec conditioning MVC, depression of the CMEP was present when stimulation occurred at rest (Fig. $3 B$, open boxes) $(p<0.05)$ and during weak brief contractions (filled boxes) $(p<0.05)$. In biceps, slightly less depression of the CMEP occurred during the weak contractions than at rest (Fig. $3 B, 0-30 \mathrm{sec})(t$ test; $p=0.026)$. In contrast, Figure $3 C$ shows that the depression was absent when assessed during brief maximal voluntary efforts (filled boxes), although its time course was unaffected when tested at rest after the MVCs (open boxes). Thus, depression of the CMEP is not apparent when tested during strong voluntary activation of the corticospinal system, although it is evident during weak activation. It should also be noted that, during the intermittent MVCs, voluntary EMG activity in biceps and brachioradialis was unchanged by the conditioning contraction. This suggests that motoneuronal output in these maximal voluntary efforts was not reduced.

\section{Psychophysical study}

Given that the depression of the CMEP probably results from presynaptic factors and that voluntary activity can "overcome" the depression, one explanation for its continued presence despite weak voluntary activity could be that CMEPs tested during the brief 5\% MVC result from stimulation of descending fibers that are not active in the contraction. Alternatively, the abolition of the depression might depend on the level of activity in each axon, so that a little activity has only a small effect on the depression, but a sufficiently large amount of activity overcomes it. If depression of corticospinal efficacy recovers only partially with weak voluntary contractions, and the relevant corticospinal synapses are used in voluntary contractions, then normal voluntary movements may be affected.

In the last series of experiments, subjects made sets of "identical" contractions (10\% MVC) with elbow flexors bilaterally and received feedback of force produced on the left side only. The subjects performed repeated elbow flexions of the same size with both arms simultaneously at $5 \mathrm{sec}$ intervals. The profiles of force in individual contractions on the right and left sides were very similar in timing and magnitude (Fig. 4). This concordance in the force of the two arms suggests that subjects sent similar commands to both sides. After a conditioning $10 \mathrm{sec} \mathrm{MVC}$ of the right arm only, the force and EMG activity produced by the right were transiently reduced. EMG activity in right elbow flexor muscles of one subject is illustrated in Figure 5A. For the group of subjects (Fig. $5 B$ ), the EMG activity was reduced for at least $30 \mathrm{sec}$ after the conditioning MVC in brachioradialis but not biceps brachii ( $p<$ 0.05). Significant reductions in EMG activity occurred at this time for 8 of 10 subjects in brachioradialis, biceps brachii, or both muscles. This reduction of activity occurred despite the increase in the size of the muscle action potential, and it did not occur when subjects rested for $10 \mathrm{sec}$ rather than performed an MVC.

\section{Discussion}

Findings from a range of experimental preparations demonstrate that the release of transmitter from a synapse depends on its preceding activity (Del Castillo and Katz, 1954; Betz, 1970; Varela et al., 1997; Stevens and Wesseling, 1998; Dittman et al., 2000) (for review, see Zucker, 1989; O'Donovan and Rinzel, 1997; Wu and Saggau, 1997; Zucker and Regehr, 2002). This applies to the cortex, the neuromuscular junction, and spinal motor circuits (Davis et al., 1985; Lev-Tov and Pinco, 1992; Hultborn et al., 1996; Parker and Grillner, 2000). Knowledge of the importance of such variations in synaptic strength in human motor control is limited. We present evidence that synaptic transmission in the human CNS depends on previous and current activity and also that this dependence affects motor behavior.

When subjects made bilateral elbow flexions after a $10 \mathrm{sec}$ MVC with the right arm, force output and EMG activity were reduced in the muscles that had performed the conditioning MVC. Whereas a reduction in force might result from fatigue of the muscles themselves, the reduction in EMG activity indicates a more central deficit. Motoneuronal output was reduced compared with before the conditioning contraction and compared 
with that on the contralateral side. During human voluntary movements, motoneurons are actively driven by cortical cells (Davey et al., 1994; Petersen et al., 2001b), but inputs from peripheral receptors also contribute, as indicated by the reduction in motoneuron firing rates with acute unloading or deafferentation (Gandevia et al., 1990; Macefield et al., 1993; Sinkjaer et al., 2000). It is unlikely that the firing of muscle spindles or tendon organs during small voluntary contractions would be altered substantially by a conditioning MVC (Vallbo, 1974; Gregory et al., 2002). Thus, the reduced EMG activity after the conditioning maximal effort may reflect a reduced activation of motoneurons from descending pathways because of altered efficacy of the corticospinal connections.

Muscle responses to a single descending volley in the corticospinal tract also tested after a $10 \mathrm{sec}$ MVC also show a long-lasting depression that we suggest results from decreased synaptic efficacy (Fig. 1) (Gandevia et al., 1999). In producing these muscle responses (CMEPs), corticospinal axons are activated at the pyramidal decussation (Ugawa et al., 1991; Gandevia et al., 1999; Taylor et al., 2002), and excitation reaches the motoneurons through one synapse (corticomotoneuronal pathway) or more than one synapse (i.e., via interneuronal pathways). In humans, there are substantial corticomotoneuronal connections to biceps brachii, and the CMEP has a large monosynaptic component (de Noordhout et al., 1999; Petersen et al., 2002; Taylor et al., 2002). For this monosynaptic pathway, factors that influence the size of the CMEP, and may depress it after a contraction, include the number of descending axons activated by the electrical stimulus, the amount of transmitter released at the presynaptic terminal, and the responsiveness of the motoneuron pool. Classical presynaptic inhibition appears not to occur at this site in humans (Nielsen and Petersen, 1994). Events at the neuromuscular junction and sarcolemma can be excluded because the muscle action potential is modestly increased after the conditioning MVCs (Fig. 2) (Cupido et al., 1996; Gandevia et al., 1999).

We argued previously that the depressed CMEP is not explained by postsynaptic changes in the motoneuronal output or gain because activation of motoneurons through antidromic invasion and afferent pathways does not depress the CMEP (Gandevia et al., 1999). In the current studies, the CMEP is depressed during a constant low level of EMG activity after a conditioning maximal contraction (Fig. 2). This suggests that the state of the motoneuronal pool does not critically affect the presence of the depression and is consistent with the view that a postsynaptic mechanism is not the primary cause of the depression (Gandevia et al., 1999).

The decrease in the CMEP could originate from an activitydependent decrease in axonal excitability. However, the depression of the CMEP is maximal after a $5 \mathrm{sec}$ MVC, with no additional depression with contractions lasting $120 \mathrm{sec}$ (Gandevia et al., 1999). Such additional activity would result in large differences in excitability of peripheral motor axons (Bergmans, 1970; Vagg et al., 1998) but has no apparent effect on the axons excited by descending tract stimulation. And as shown here, there is no depression of the CMEP tested during brief MVCs, whereas the
B

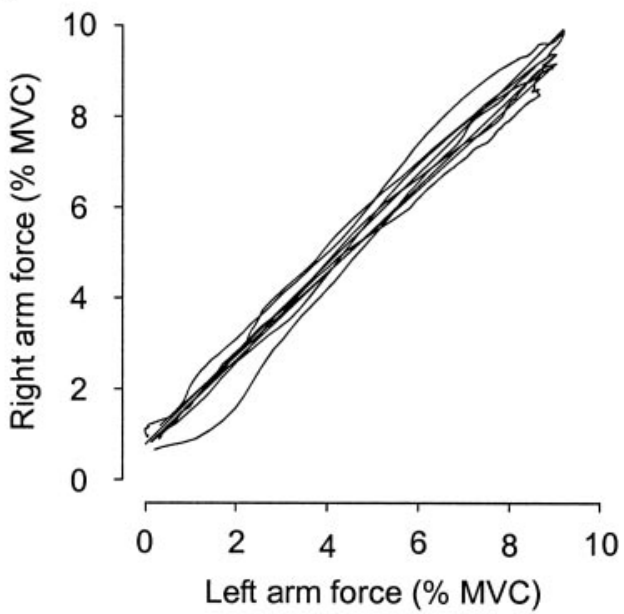

CMEP tested at rest is depressed (Fig. 3C). Depression resulting from altered axonal excitability should occur during contraction as well as at rest. Thus, we propose that the depression of the CMEP after a voluntary effort may result from altered synaptic transmission.

The present studies offer insight into the characteristics of the depression of the CMEP caused by voluntary contraction. The magnitude of depression depends on the strength of the conditioning $10 \mathrm{sec}$ contraction (Fig. 1) and is thus related to the amount of preceding activity. However, the depression does not depend on the duration of a maximal effort (provided that it exceeds $5 \mathrm{sec}$ ), and so it is not just the number of action potentials conveyed by descending axons that is important. Because recordings in the monkey reveal that cortical cells increase their firing rate with increased muscle activity and force (Evarts, 1968; Lemon et al., 1986; Fetz et al., 1989), the different strengths of conditioning contraction probably represent different firing frequencies in the corticospinal system. Hence, changes in synaptic transmission that underlie the depression of the CMEP depend on the frequency of preceding activity. A second finding is that no depression of the CMEP is apparent during brief MVCs after the conditioning contraction, whereas CMEPs tested at rest show depression. At this time, CMEPs are also depressed during weak brief contractions. Thus, the long-lasting depression can be temporarily overcome by sufficient descending activity, and this temporary "recovery" may also depend on the frequency of activity.

Individual synaptic processes, with time courses that vary from milliseconds to minutes, can simultaneously facilitate and depress the release of transmitter and could underlie the depression reported here (Zucker, 1989; Wu and Saggau, 1997; Zucker and Regehr, 2002). If a synapse is driven at a sufficiently high rate for sufficient time, the synaptic vesicles in the pool available for immediate release eventually deplete. Homosynaptic inhibitory mechanisms may also reduce release (Redman and Silinsky, 1994). A frequency-dependent depletion that is maximal after 5 sec of maximal voluntary activity could explain the depression of the CMEP. The transient amelioration of the depression during brief MVCs suggests involvement of a second process, such as one that controls the turnover of releasable vesicles during repetitive activation. Enhancements that are graded with activity have been 


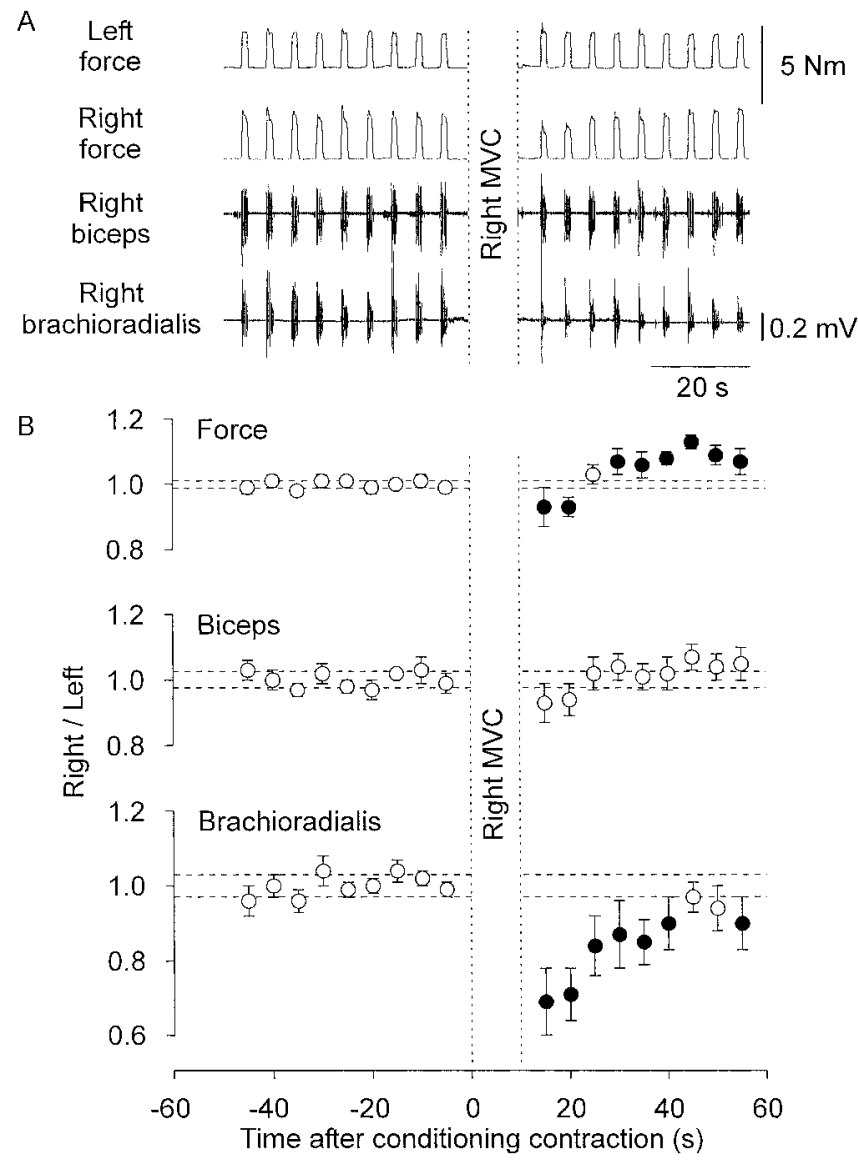

Figure 5. Psychophysical experiment that reveals a reduced output on the right side after a $10 \mathrm{sec}$ MVC on that side. $A$, One set of contractions in a single subject. The subject produced simultaneous elbow flexion contractions to a target force on the left (10\% MVC). Traces show left and right elbow flexion forces and EMG activity from right biceps brachii and brachioradialis. After a 10 sec MVC on the right, lower force and EMG activity were produced on the right. $B$, Pooled data (mean $\pm \mathrm{SEM} ; n=10$ ) for the matching study. Data are shown for the nine contractions before and after a 10 sec MVC on the right. Force, biceps brachii EMG activity, and brachioradialis EMG activity are plotted as a ratio between the right and left (control) side, with the horizontal lines showing \pm 1 SD. The values were normalized to the mean for the nine control contractions before the MVC. The ratio for brachioradialis EMG activity was depressed after the conditioning MVC, and it recovered over $\sim 40 \mathrm{sec}$. This depression of the EMG activity occurred despite the likely increase in the muscle fiber action potential in active fibers (Fig. $2 \mathrm{C}$ (Cupido et al., 1996; Gandevia et al., 1999). Significant changes from control at times after the MVC are shown as filled circles $(p<0.05)$. The open circles after the conditioning contraction indicate data not significantly changed.

reported at several types of synapses (Hochner et al., 1986; Kuromi and Kidokoro, 1998; Delgado et al., 2000; Dittman et al., 2000; Pyle et al., 2000; Richards et al., 2000; Beutner et al., 2001). Such processes would be needed for repetitive or sustained voluntary contractions at high intensities.

A decrease in synaptic transmission that has the characteristics defined by the behavior of the CMEP should affect weak voluntary contractions and could explain the findings of the psychophysical study. Subjects performed consistent bilateral elbow flexions in a task designed to keep constant the motor cortical output that produced the weak contractions. If the motor cortical output remained the same, then reduced synaptic efficacy after the conditioning contraction should activate fewer motoneurons. We saw this as reduced EMG activity in the muscle that had performed the conditioning MVC. In contrast, when subjects performed intermittent brief maximal efforts, there was no re- duction in EMG activity and the CMEP depression was transiently overcome. If a constant submaximal level of EMG activity was required, output from the motor cortex could be increased to overcome any synaptic depression, and subjects might perceive an increase in the "effort" needed for the contraction.

Although "depression" of EMG activity occurred in the psychophysical experiment, it was less than when the corticospinal system was tested with a single electrical stimulus. One factor contributing to this difference could be that activity in motoneurons during voluntary contraction is influenced by many sources, e.g., corticospinal, noncorticospinal, and afferent pathways. Therefore, synaptic depression at the corticospinal synapses will appear less obvious in the overall activity of the motoneurons than in the response to a single corticospinal volley. Additionally, the target force for the weak contractions was $10 \%$ MVC compared with rest or $5 \%$ MVC during which CMEPs were examined. Even this low level of activity may partially overcome the depression.

Our results on the depression of the response to a corticospinal stimulus after strong voluntary activity are consistent with the presence of activity-dependent changes at corticospinal synapses. Such changes may both depress and facilitate synaptic efficacy. Given the plasticity of synaptic behavior reported at many sites in the CNS, our proposal simply recognizes that this plasticity probably applies to the major pathway for voluntary movement. If so, it points to the need for proprioceptive feedback to regulate the descending input to muscles and to the need for synaptic processes that counteract any depression and thus allow repetitive voluntary movement.

\section{References}

Bergmans J (1970) The physiology of single human nerve fibres. Vander, Belgium: University of Louvain.

Betz WJ (1970) Depression of transmitter release at the neuromuscular junction of the frog. J Physiol (Lond) 206:629-644.

Beutner D, Voets T, Neher E, Moser T (2001) Calcium dependence of exocytosis and endocytosis at the cochlear inner hair cell afferent synapse. Neuron 29:681-690.

Cupido CM, Galea V, McComas AJ (1996) Potentiation and depression of the M wave in human biceps brachii. J Physiol (Lond) 491:541-550.

Davey NJ, Romaiguere P, Maskill DW, Ellaway PH (1994) Suppression of voluntary motor activity revealed using transcranial magnetic stimulation of the motor cortex in man. J Physiol (Lond) 477:223-235.

Davis BM, Collins III WF, Mendell LM (1985) Potentiation of transmission at Ia-motoneuron connections induced by repeated short bursts of afferent activity. J Neurophysiol 54:1541-1552.

de Noordhout AM, Rapisarda G, Bogacz D, Gerard P, De Pasqua V, Pennisi G, Delwaide PJ (1999) Corticomotoneuronal synaptic connections in normal man: an electrophysiological study. Brain 122:1327-1340.

Del Castillo J, Katz B (1954) Statistical factors involved in neuromuscular transmission. J Physiol (Lond) 124:574-585.

Delgado R, Maureira C, Oliva C, Kidokoro Y, Labarca P (2000) Size of vesicle pools, rates of mobilization, and recycling at neuromuscular synapses of a Drosophila mutant, shibire. Neuron 28:941-953.

Dittman JS, Kreitzer AC, Regehr WG (2000) Interplay between facilitation, depression, and residual calcium at three presynaptic terminals. J Neurosci 20:1374-1385.

Evarts EV (1968) Relation of pyramidal tract activity to force exerted during voluntary movement. J Neurophysiol 31:14-27.

Fetz EE, Cheney PD, Mewes K, Palmer S (1989) Control of forelimb muscle activity by populations of corticomotoneuronal and rubromotoneuronal cells. Prog Brain Res 80:437-449.

Fisher SA, Fischer TM, Carew TJ (1997) Multiple overlapping processes underlying short-term synaptic enhancement. Trends Neurosci 20:170-177.

Gandevia SC, Macefield G, Burke D, McKenzie DK (1990) Voluntary activation of human motor axons in the absence of muscle afferent feedback. The control of the deafferented hand. Brain 113:1563-1581.

Gandevia SC, Petersen N, Butler JE, Taylor JL (1999) Impaired response of 
human motoneurones to corticospinal stimulation after voluntary exercise. J Physiol (Lond) 521:749-759.

Gregory JE, Brockett CL, Morgan DL, Whitehead NP, Proske U (2002) Effect of eccentric muscle contractions on Golgi tendon organ responses to passive and active tension in the cat. J Physiol (Lond) 538:209-218.

Hochner B, Klein M, Schacher S, Kandel ER (1986) Additional component in the cellular mechanism of presynaptic facilitation contributes to behavioral dishabituation in Aplysia. Proc Natl Acad Sci USA 83:8794-8798.

Hultborn H, Illert M, Nielsen J, Paul A, Ballegaard M, Wiese H (1996) On the mechanism of the post-activation depression of the H-reflex in human subjects. Exp Brain Res 108:450-462.

Kuromi H, Kidokoro Y (1998) Two distinct pools of synaptic vesicles in single presynaptic boutons in a temperature-sensitive Drosophila mutant, shibire. Neuron 20:917-925.

Lemon RN, Mantel GW, Muir RB (1986) Corticospinal facilitation of hand muscles during voluntary movement in the conscious monkey. J Physiol (Lond) 381:497-527.

Lev-Tov A, Pinco M (1992) In vitro studies of prolonged synaptic depression in the neonatal rat spinal cord. J Physiol (Lond) 447:149-169.

Macefield VG, Gandevia SC, Bigland-Ritchie B, Gorman RB, Burke D (1993) The firing rates of human motoneurones voluntarily activated in the absence of muscle afferent feedback. J Physiol (Lond) 471:429-443.

Miller RJ (1998) Presynaptic receptors. Annu Rev Pharmacol Toxicol 38:201-227.

Muir RB, Porter R (1973) The effect of a preceding stimulus on temporal facilitation at corticomotoneuronal synapses. J Physiol (Lond) 228:749-763.

Nielsen J, Petersen N (1994) Is presynaptic inhibition distributed to corticospinal fibres in man? J Physiol (Lond) 477:47-58.

O'Donovan MJ, Rinzel J (1997) Synaptic depression: a dynamic regulator of synaptic communication with varied functional roles. Trends Neurosci 20:431-433.

Parker D, Grillner S (2000) The activity-dependent plasticity of segmental and intersegmental synaptic connections in the lamprey spinal cord. Eur J Neurosci 12:2135-2146.

Petersen NT, Taylor JL, Gandevia SC (2001a) Stimulation of the corticospinal tract activates descending neurones with direct connections to human motoneurones. IV International Congress of Physiological Sciences: CD Rom 1853.

Petersen NT, Butler JE, Marchand-Pauvert V, Fisher R, Ledebt A, Pyndt HS, Hansen NL, Nielsen JB (2001b) Suppression of EMG activity by transcranial magnetic stimulation in human subjects during walking. J Physiol (Lond) 537:651-656.

Petersen NT, Taylor JL, Gandevia SC (2002) The effect of electrical stimulation of the corticospinal tract on motor units of human biceps brachii. J Physiol (Lond) 544:277-284.

Phillips CG, Porter R (1977) Corticospinal neurones: their role in movement. London: Academic.
Porter R (1970) Early facilitation at corticomotoneuronal synapses. J Physiol (Lond) 207:733-745.

Porter R, Lemon RN (1993) Corticospinal function and voluntary movement. Oxford: Clarendon.

Pyle JL, Kavalali ET, Piedras-Renteria ES, Tsien RW (2000) Rapid reuse of readily releasable pool vesicles at hippocampal synapses. Neuron 28:221-231.

Redman RS, Silinsky EM (1994) ATP released together with acetylcholine as the mediator of neuromuscular depression at frog motor nerve endings. J Physiol (Lond) 477:117-127.

Richards DA, Guatimosim C, Betz WJ (2000) Two endocytic recycling routes selectively fill two vesicle pools in frog motor nerve terminals. Neuron 27:551-559.

Shapovalov AI, Shiriaev BI, Velumian AA (1978) Mechanisms of postsynaptic excitation in amphibian motoneurones. J Physiol (Lond) 279:437-455.

Sinkjaer T, Andersen JB, Ladouceur M, Christensen LO, Nielsen JB (2000) Major role for sensory feedback in soleus EMG activity in the stance phase of walking in man. J Physiol (Lond) 523:817-827.

Stevens CF, Wesseling JF (1998) Activity-dependent modulation of the rate at which synaptic vesicles become available to undergo exocytosis. Neuron 21:415-424.

Taylor JL, Petersen N, Butler JE, Gandevia SC (2000) Ischaemia after exercise does not reduce responses of human motoneurones to cortical or corticospinal tract stimulation. J Physiol (Lond) 525:793-801.

Taylor JL, Petersen NT, Butler JE, Gandevia SC (2002) Interaction of transcranial magnetic stimulation and electrical transmastoid stimulation in human subjects. J Physiol (Lond) 541:949-958.

Thomson AM (2000) Facilitation, augmentation and potentiation at central synapses. Trends Neurosci 23:305-312.

Ugawa Y, Rothwell JC, Day BL, Thompson PD, Marsden CD (1991) Percutaneous electrical stimulation of corticospinal pathways at the level of the pyramidal decussation in humans. Ann Neurol 29:418-427.

Vagg R, Mogyoros I, Kiernan MC, Burke D (1998) Activity-dependent hyperpolarization of human motor axons produced by natural activity. J Physiol (Lond) 507:919-925.

Vallbo AB (1974) Human muscle spindle discharge during isometric voluntary contractions. Amplitude relations between spindle frequency and torque. Acta Physiol Scand 90:319-336.

Varela JA, Sen K, Gibson J, Fost J, Abbott LF, Nelson SB (1997) A quantitative description of short-term plasticity at excitatory synapses in layer $2 / 3$ of rat primary visual cortex. J Neurosci 17:7926-7940.

Wu LG, Saggau P (1997) Presynaptic inhibition of elicited neurotransmitter release. Trends Neurosci 20:204-212.

Zucker RS (1989) Short-term synaptic plasticity. Annu Rev Neurosci 12:13-31.

Zucker RS, Regehr WG (2002) Short-term synaptic plasticity. Annu Rev Physiol 64:355-405. 